\title{
BMJ Open Investigating the natural history and prognostic factors of ASD in children: the multicEntric Longitudinal study of childrEN with ASD - the ELENA study protocol
}

\begin{abstract}
Amaria Baghdadli, ${ }^{1,2}$ Stéphanie Miot, ${ }^{1,2}$ Cécile Rattaz, ${ }^{1}$ Tasnime Akbaraly, ${ }^{1,3}$ Marie-Maude Geoffray, ${ }^{4}$ Cécile Michelon, ${ }^{1}$ Julie Loubersac, ${ }^{1}$ Sabine Traver, ${ }^{1}$ Marion Mortamais, ${ }^{1}$ Sandrine Sonié, ${ }^{5,6}$ Julien Pottelette, ${ }^{7}$ Laurence Robel, ${ }^{8}$ Mario Speranza, ${ }^{9,10}$ Stéphanie Vesperini, ${ }^{11}$ Thierry Maffre, ${ }^{12}$ Bruno Falissard, ${ }^{2}$ Marie-Christine Picot, ${ }^{13}$ for the ELENA Study Group
\end{abstract}

To cite: Baghdadli A, Miot S, Rattaz C, et al. Investigating the natural history and prognostic factors of ASD in children: the multicEntric Longitudinal study of childrEN with ASD - the ELENA study protocol. BMJ Open 2019;9:e026286. doi:10.1136/ bmjopen-2018-026286

- Prepublication and additional material are published online only. To view please visit the journal online (http://dx.doi. org/10.1136/bmjopen-2018026286).

Received 24 August 2018 Revised 11 January 2019 Accepted 8 May 2019

\section{Check for updates}

(C) Author(s) (or their employer(s)) 2019. Re-use permitted under CC BY-NC. No commercial re-use. See rights and permissions. Published by BMJ.

For numbered affiliations see end of article.

\section{Correspondence to} Prof. Amaria Baghdadli; rech-clinique-autisme@chumontpellier.fr

\begin{abstract}
Introduction There is global concern about the increasing prevalence of autism spectrum disorders (ASDs), which are early-onset and long-lasting disorders. Although ASDs are considered to comprise a unique syndrome, their clinical presentation and outcome vary widely. Large-scale and long-term cohort studies of well-phenotyped samples are needed to better understand the course of ASDs and their determinants. The primary objective of the multicEntric Longitudinal study of childrEN with ASD (ELENA) study is to understand the natural history of ASD in children and identify the risk and prognostic factors that affect their health and development.
\end{abstract}

Methods and analysis This is a multicentric, longitudinal, prospective, observational cohort in which 1000 children with ASD diagnosed between 2 and 16 years of age will be recruited by 2020 and followed over 6 years. The baseline follow-up starts with the clinical examination to establish the ASD diagnosis. A battery of clinical tools consisting of the Autism Diagnostic Observation Schedule, the revised version of the Autism Diagnostic Interview, measures of intellectual functioning, as well as large-scale behavioural and developmental measurements will allow us to study the heterogeneity of the clinical presentation of ASD subtypes. Subsequent follow-up at 18 months and at $3,4.5$ and 6 years after the baseline examination will allow us to explore the developmental trajectories and variables associated with the severity of ASD. In addition to the children's clinical and developmental examinations, parents are invited to complete self-reported questionnaires concerning perinatal and early postnatal history, congenital anomalies, genetic factors, lifestyle factors, medical and psychiatric comorbidities, and the socioeconomic environment. As of 1 November 2018, a total of 766 participants have been included.

Ethics and dissemination Ethical approval was obtained through the Marseille Mediterranean Ethics Committee (ID RCB: 2014-A01423-44), France. We aim to disseminate the findings through national and international conferences, international peer-reviewed journals, and social media.
Strengths and limitations of this study

- This study will constitute one of the largest studies of children with accurate diagnoses of autism spectrum disorder (ASD) prospectively followed for 6 years to assess their outcome trajectories.

- It will simultaneously assess multiple domains, enabling an indepth analysis of the natural history of ASD and its clinical heterogeneity, and examination of the prognostic factors.

- The representative nature of our sample is limited as children are being recruited in autism centres.

Trial registration number NCT02625116; Pre-results.

\section{INTRODUCTION}

Autism spectrum disorders (ASDs), which affect $1 \%$ of children and adults, are neurodevelopmental disorders typically diagnosed in early childhood, with a higher prevalence rate among males than females. ${ }^{1}$ They are characterised by deficits in social communication associated with a pattern of repetitive and stereotyped behaviours and interests/ activities, of which the clinical presentation is highly heterogeneous. ${ }^{23}$ The causes are still unknown, but are believed to be complex and multifactorial, associated with both genetic and environmental factors that probably dynamically interact during critical time windows, especially the prenatal and early postnatal periods. ${ }^{45}$ ASDs are a major cause of morbidity and disability, as they begin in very early life, are long-lasting, and ${ }^{67}$ often associated with many other chronic medical 
conditions that may also have a major negative impact on functioning and outcome. ${ }^{89}$

Although there is no cure for ASD, targeted behavioural and developmental interventions are employed, but the evidence is still limited concerning how, when and for whom their efficacy is optimal among the large spectrum of individuals with autism. ${ }^{10}$ The family burden is important and leads to higher stress and a lower quality of life (QoL) than for other chronic conditions, but the impact is highly variable depending on the clinical phenotype. ${ }^{11-14}$ The economic and societal impact associated with ASD is also substantial and growing. Thus adequate care, well-adapted support structures for affected individuals and their families, and effective treatment to improve daily functioning and outcomes are crucial. ${ }^{15}$

The extreme heterogeneity in clinical presentation and outcome is one of the most frequently raised issues by families and policy makers. It is also one of the most striking and puzzling aspects of ASD, in terms of overall independence and changes in autistic symptoms and behaviours over time, and in terms of aetiologies and personalised medicine. ${ }^{16}$ The marked heterogeneity of ASDs has led to suggestions that rather than being considered a single disorder, it might be constructive to reframe ASDs as 'the autisms', thereby giving consideration to multiple aetiologies and distinct clinical entities. ${ }^{17} 18$

Although the heterogeneity of ASD, including genetic variability and comorbidity, is well established, ${ }^{19}$ knowledge concerning the developmental course of symptom expression that defines ASD and the determinants of the diverse trajectories is still limited, as most longitudinal studies are limited by retrospective design, short time frames and infrequent follow-up of the populations. ${ }^{719-21}$ In addition, even though there is a significant body of evidence that predictors of autism exist in early childhood, probably in part as a function of the multiple genes involved, the myriad of environmental factors and the co-occurrence of medical and mental health dysfunctions, small sample size, and high age at recruitment still limit the general predictive and clinical value of such parameters.

Examining and deconstructing the heterogeneity of outcome trajectories represent both a promising route and a challenge for our understanding of the causes of ASD and the development of biomarkers of risk, prediction and stratification. ${ }^{22}$ Recent studies attempting to map developmental trajectories in ASD have been published over the last few years but, with rare exceptions, ${ }^{23}{ }^{24}$ most did not combine a large sample of young children, multidimensional data collection and long-term follow-up..$^{20-27}$ Thus, it has been stressed that studies have to be longitudinal, prospective and multicentre, with large and multidimensional-based data collection, and the recent trend has been to move the targeted times of enrolment to very early in childhood. ${ }^{28}$

Our study aims to create, examine and prospectively follow up a large cohort of children, who live in France, with an ascertained ASD diagnosis, evaluating the long-term natural history of ASD features to further identify patterns of outcome trajectories associated with multiple clinical, developmental, behavioural, environmental and social variables. We are specifically interested in providing a multidimensional analysis of the predictors of the heterogeneity of the long-term outcome trajectories of children with ASD. Such a study has a high potential to influence national policies in many aspects of healthcare, health promotion and disease prevention, and ultimately the well-being of the population.

\section{METHODS AND ANALYSIS}

\section{Study design}

This is a multicentric, ongoing, prospective longitudinal cohort study.

\section{Study setting}

This study is taking place in France and involves $50 \%$ of all French autism research centres $(n=13)$. According to national guidelines, ASD diagnosis has to be based on an exhaustive multidisciplinary process. It is mainly achieved in France in secondary settings, but with inconsistencies in practices across areas. For these reasons, the recruitment was planed from tertiary centres. These centres, spread throughout the French territories, are specialised in the assessment and diagnosis of ASD and other neurodevelopmental disorders and respectful of best practices guidelines. Children were ordinarily referred from primary or secondary care by their family doctor, child psychiatrist or paediatrician. Recruitment started in 2012 and will continue until 2020 to include 1000 participants.

\section{Study population}

The inclusion criteria are participants between 2 and 16 years of age, referred for a first diagnostic established by a multidisciplinary clinical assessment to one of the 13 autism research centres, diagnosed with pervasive developmental disorders (PDDs) or ASD according to the International Classification of Diseases (ICD) 10 (which was recommended in France at the onset of the study $^{29}{ }^{30}$ ), and whose parents read and speak French. After the publication in France in 2014 of the Diagnostic and Statistical Manual of Mental Disorders (DSM-5), all the children were diagnosed with this classification, and those diagnosed before as PDD were recoded using DSM-5.

The exclusion criterion is parental refusal.

\section{Patient selection, recruitment and initial assessment}

Participants were recruited nationally in public settings from tertiary centres in the course of a multidisciplinary assessment. After ascertaining patient eligibility, information concerning the study protocol was given to parents during a medical consultation by the autism research centre investigator and their written consent was collected. Parents/caregivers who agreed to participate received online questionnaires about their child. 


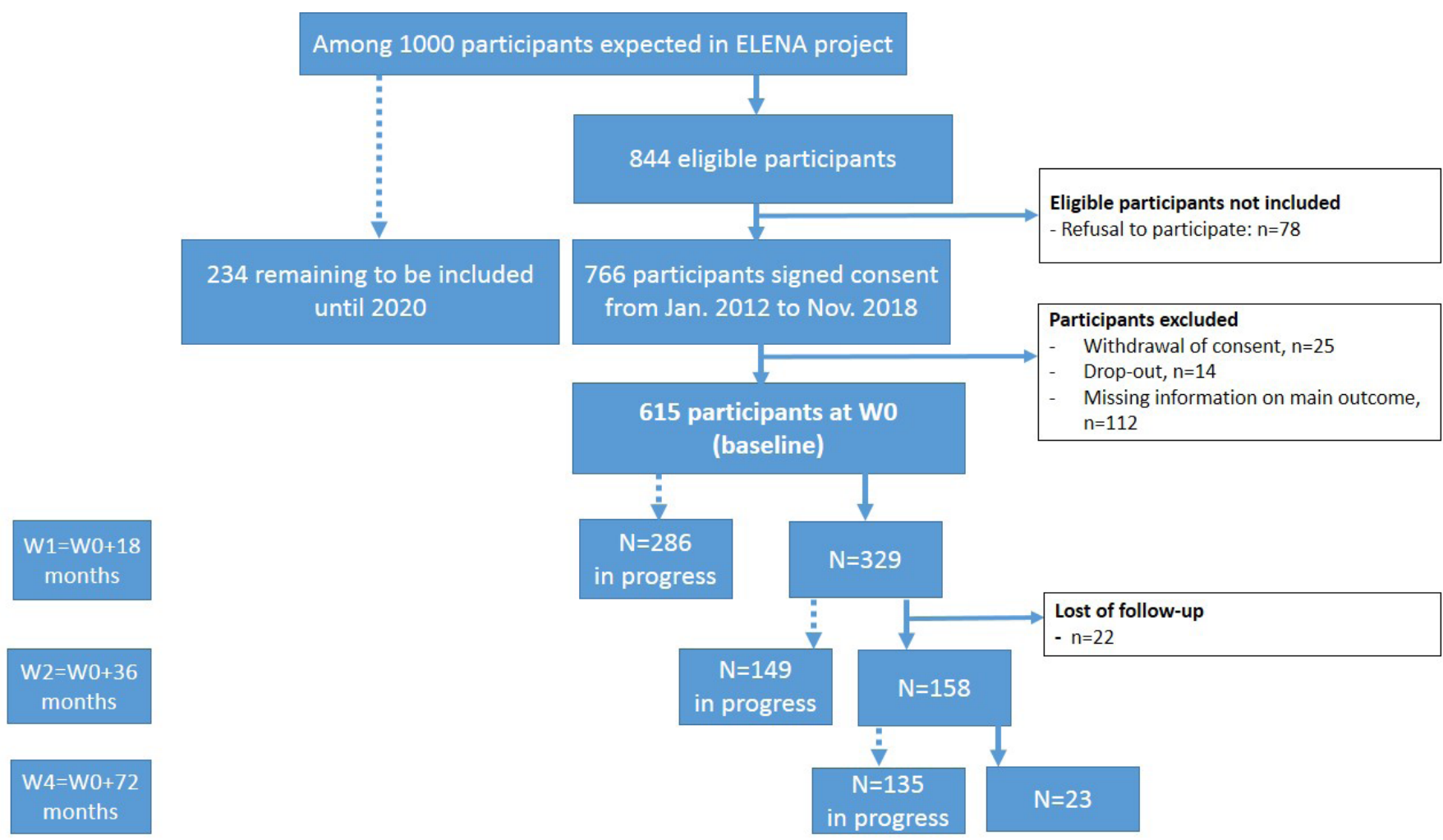

Figure 1 Flow chart of the 615 children from the ongoing open ELENA cohort. ELENA, multicEntric Longitudinal study of childrEN with ASD; W, wave.

As of 1 November 2018, 844 children were eligible to participate in the study and written parental consent was obtained for 766 (acceptation rate: 91\%). A flow chart describing the study protocol and selection of the children participating in the multicEntric Longitudinal study of childrEN with ASD (ELENA) study is shown in figure 1. The geographical breakdown of the included participants is uneven (illustrated in online supplementary figure A), with most of the cases recruited from the south of France.

\section{Follow-up assessments}

Following the baseline clinical examination (wave 0), subsequent follow-ups take place at 18 months (W1) and at 3 (W2), 4.5 (W3) and 6 (W4) years after the baseline examination, consisting of alternate postal/online questionnaires alone (W1 and W3) and postal questionnaires and clinical examinations (W2 and W4) to assess the evolution of pervasive clinical and behavioural symptoms of the disorder. At each visit (W2 and W4), a clinical report summarising the results of the assessments and the clinician's analysis concerning the child's outcome since the previous visit is provided to parents and local healthcare or educational services designated by the parents.

\section{Study outcomes}

The primary study outcome consists of the scores for the three domains of the Vineland Scale (communication, socialisation and daily living skills) as highly correlated with intellectual measures and adequately translating the individual's cognitive abilities in real life. ${ }^{31-33}$ The Vineland Scale can be used in all children for each monitoring of the follow-up, even in those where cognitive tests cannot be performed. ${ }^{34}$

The secondary outcomes include autistic symptoms, sensory profile, language level, intellectual functioning, individual and familial QoL, parental stress, and school achievement. Autistic status was also reviewed and discussed by a committee of trained clinicians (child psychiatrists, psychologists and speech therapist) to obtain a consensus on the ASD diagnosis according to the DSM-5 criteria.

\section{Data collection and study measures}

Data are collected by licensed psychologists from the multidisciplinary clinical assessments using standardised tools at baseline and at each follow-up visit (detailed in table 1). The medical data are completed directly online at every site on a data entry form created via specific software (Ennov Clinical). This software respects the recommendations of the Food and Drug Administration (FDA) concerning informatics systems for the management of clinical trials and standards (clinical data interchange standards consortium (CDISC), International Conference on Harmonization (ICH), good clinical practice (GCP) 2001/20/CE). In addition, parents complete online self-reported questionnaires. All data are anonymous and personally identifiable information is stored separately from the other data.

The ASD diagnostic process is based on a two-step procedure. First, a certified child psychiatrist or psychologist administers a battery of four clinical tools consisting 
Table 1 Measurements of the ELENA study at the four timepoints of the follow-up

\section{Measurement}

\section{Direct clinical observation of the child Assessment}

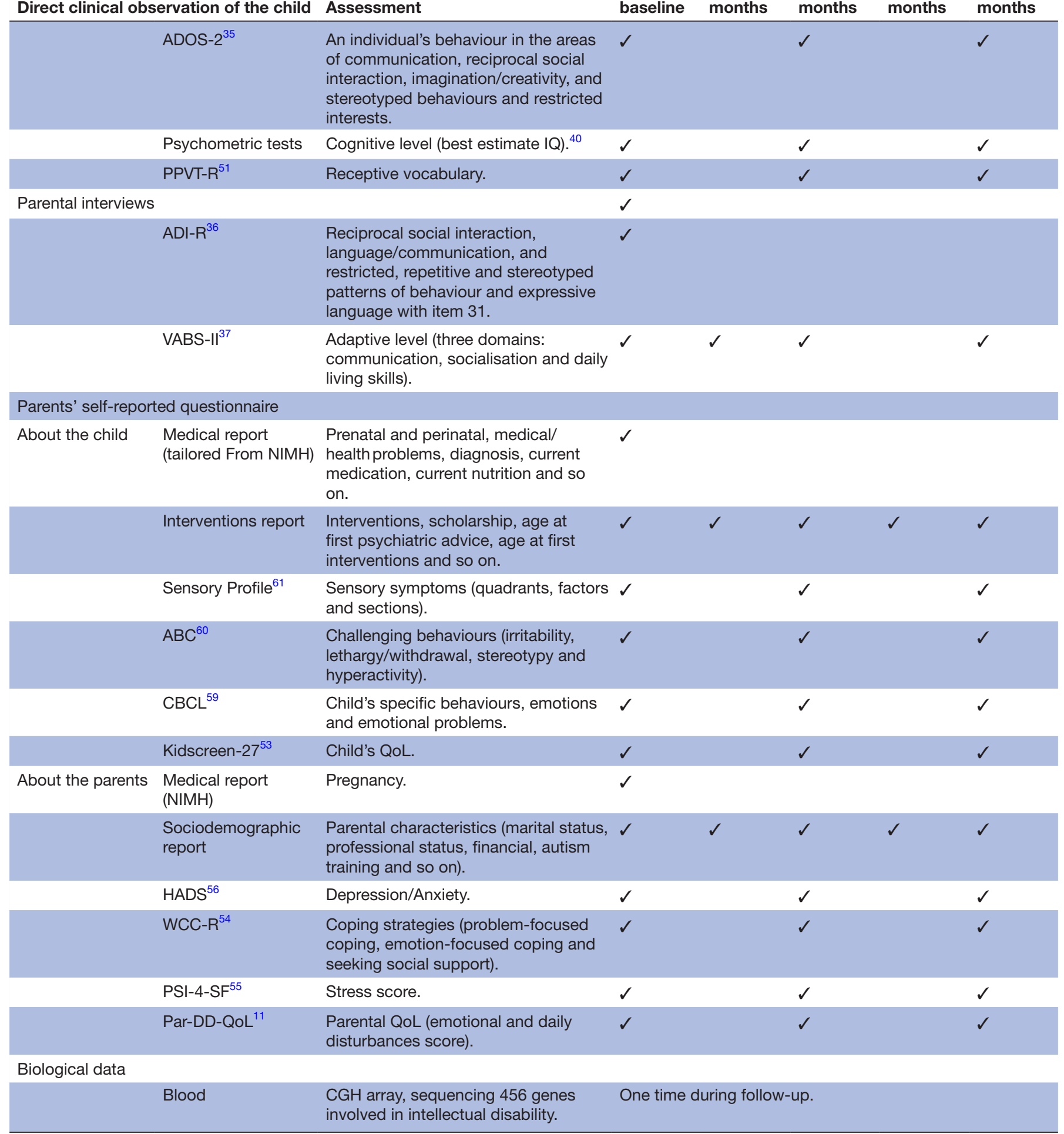

ABC, Aberrant Behavior Checklist; ADI-R, revised version of the Autism Diagnostic Interview; ADOS-2, Autism Diagnostic Observation Schedule; ASD, autism spectrum disorder; CBCL, Child Behavior Checklist; $\mathrm{CGH}=$ Comparative genomic hybridization; ELENA, multicEntric Longitudinal study of childrEN with ASD; HADS, Hospital Anxiety and Depression Scale; NIMH, Nationallnstitute of Mental Health; Par-DDQoL, Parental-Developmental Disorder-Quality of Life; PPVT-R, Peabody Picture Vocabulary Test-Revised; PSI-4-SF, Parenting Stress Index, Fourth Edition Short Form; QoL, quality of life; VABS-II, Vineland Adaptive Behavior Scales, Second Edition W, wave; WCC-R, French Ways of Coping Checklist. 
of the Autism Diagnostic Observation Schedule (ADOS2), ${ }^{35}$ the revised version of the Autism Diagnostic Interview (ADI-R) ${ }^{36}$ a best estimate of the intellectual functioning level and the Vineland Adaptive Behavior Scales, Second Edition (VABS-II) ${ }^{37}$ (all items are detailed below). Second, a committee of trained clinicians and child psychiatrists or psychologists discuss and review all cases to obtain a consensus on the ASD diagnosis (at baseline and follow-up) according to the ICD or DSM- 5 criteria, which were the recommended classification in France until 2018. ${ }^{38}$ The ASD diagnostic tools, all available in French version and used in previous French prospective cohorts, are as follows:

- The ADOS-2 $2^{35}$ is a semistructured, standardised questionnaire that assesses four domains of the individual's behaviour: (1) communication, (2) reciprocal social interaction, (3) imagination/creativity, and (4) stereotyped behaviours and restricted interests. Beyond the ASD diagnosis, ADOS provides a measure of the severity of the symptoms encompassing the (1) social affect (involving communication and social interaction items) and (2) restricted and repetitive behaviour (involving repetitive and stereotyped behaviour items). Based on this assessment, a 10-point ADOS severity score is determined for each participant. ${ }^{35} 39$

- The ADI- $\mathrm{R}^{36}$ is a standardised semistructured parent/ caregiver interview. It consists of 93 items and explores the child's early development, acquisition of language skills, language and communication functioning, loss of both speech and motor skills, social development and play, interests, and behaviour. Three scores are derived to assess abilities in communication, social reciprocity, and restricted and repetitive behaviour. A fourth score that assesses expressive language and speech is derived but does not enter into the ASD diagnosis clinical tools.

In addition to the ASD clinical tools, the clinical examination of the child includes the following:

The best estimate of intellectual level (defined as the ratio of the developmental age scores on age and multiplied by 100) is based on an age-appropriate test according to the approach of Howlin et al. ${ }^{40}$ An intellectual quotient is derived from the Wechsler Intelligence Scales ${ }^{4-46}$ and Kaufman Assessment Battery for Children. ${ }^{47}$ When it is not possible to derive an intellectual quotient (when the participants cannot understand the test instructions or requirements), a developmental quotient is determined based on revised psychoeducational profile ${ }^{48}$ and developmental scales. ${ }^{450}$

VABS-II ${ }^{37}$ is a standardised semistructured interview administered to parents to evaluate children's adaptive skills. From this questionnaire, three subscores are commonly derived that assess the child's abilities in communication, daily living skills and socialisation. The VABS-II evaluation has already been performed in a subsample of the study at 18, 36 and 72 months of follow-up ( $\mathrm{n}=329$ at 18 months, $\mathrm{n}=158$ at 36 months and $\mathrm{n}=23$ at 72 months of follow-up as of 1 November 2018; see figure 1).

Child language assessment: (1) The Peabody Picture Vocabulary Test-Revised ${ }^{51} 52$ comprised 170 items designed to evaluate the general receptive vocabulary of children and adolescents. It is administered by speech therapists with no time limit. For each item of the test, the examiner says a word and the participant then selects the corresponding picture from among four pictures presented on a page. (2) Expressive language and speech are clinically described according to three levels: (a) spontaneous and functional speech with sentences, (b) utterances or phrase speech, including at least five different words, and (c) the use of fewer than five words measured using item 'number 19' of ADI-R.

In addition to the children's clinical and developmental examinations, parents are invited to complete web-based self-reported questionnaires including the following:

Sociodemographic information: parent's age, marital status, educational level, socioeconomic status and siblings.

Medically relevant information: pregnancy, birth and neonatal care, as well as information on early development, medical diagnoses assigned to the child and medical observations (related to prescribed medication, diet and gastrointestinal outcomes).

Intervention: age at first intervention, type of intervention, and mainstream or specialised school.

Psychosocial information concerning (1) parents' perception of their child's QoL using the Kidscreen-27 Parental Form ${ }^{53}$; (2) parents' perception of the impact of ASD on their QoL using the Parental-Developmental Disorder-Quality of Life ${ }^{11}$; ; (3) parents' coping strategies using the French Ways of Coping Checklist ${ }^{54}$ and parents' stress level directly associated with their parenting role using the Parenting Stress Index, Fourth Edition Short Form ${ }^{55}$; and (4) parents' anxiety and depression using the Hospital Anxiety and Depression Scale. ${ }^{56}$

Child's behaviour collected through standardised scales consisting of the following:

1. The Child Behavior Checklist $1-5$ and $6-18^{57-59}$ is a norm-referenced measure that assesses a wide range of emotional and behavioural disorders in children aged $1.5-5$ years and 6-18years, respectively, consisting of two broadband scales and five different DSM-oriented scales. Briefly, children in the clinical or borderline clinical range are compared with children in the normal range for each of the seven scales. The construction and cut-off for each range and each scale are detailed in online supplementary methods.

2. The Aberrant Behavior Checklist ${ }^{60}$ is a 58 -item behaviour rating scale that measures behavioural problems across four domains: irritability, lethargy/withdrawal, stereotypy and hyperactivity. For each domain, scores are computed and reduced to a scale of 100, with higher scores indicating higher levels of aberrant 
behaviour. More details are provided in online supplementary methods.

3. The Sensory Profile ${ }^{61}$ is a caregiver questionnaire of 125 items measuring sensory processing abilities. Results are presented in four quadrants: low registration, sensory seeking, sensory sensitivity and sensory avoiding. Lower scores indicate more severe sensory processing difficulties.

In addition, linkage of the ELENA study children to the anonymised French National Health Data Base will allow the group to collect data on medication, hospitalisation events and history since birth. Medical events and drug consumption during pregnancy will also be collected.

\section{Data quality control procedures}

The study is coordinated by the Autism Resources Center of Montpellier University ( $\mathrm{AB}$ is the scientific coordinator). We have implemented quality control procedures concerning training and data collection in all centres of the ELENA study. Meetings were organised in each centre before the beginning of enrolment to set up protocol procedures. We ensured that ADOS and ADI were administered and scored by qualified/certified personnel. We performed cross-site training for the administration and scoring of VABS-II. Regular phone meetings with investigators or staff involved in data collection are regularly held to discuss patient enrolment and problems encountered in conducting the study, and representatives from all centres meet in Montpellier once a year. Computerised tests have been implemented to ensure the completeness, consistency and reliability of data during data entry and before the statistical analysis. Each site investigator is responsible for their contribution to the ELENA study and is part of the ELENA group. The centres associated with the project are organised into a consortium. A steering committee and a scientific committee meet each year to ensure correct follow-up of the project.

\section{Patient and public involvement}

Based on our extensive experience of working in collaboration with patient and public involvement (PPI) representatives, we included in our scientific committee two PPI representatives. They assisted the project research team to build the research proposal by paying attention to the importance and relevance of the research question for the patient's family as well as the acceptability of the research design for patients. Parents of ASD children included in the ELENA cohort received a written summary of their child's visit results and study updates through an annual newsletter and information on the ELENA cohort website (www.elena-cohorte.org).

\section{Data analysis}

We will use latent class growth modelling methods ${ }^{62} 63$ to perform the clinical feature trajectories of children with ASD and further identify patterns of ASD clinical outcome trajectories based on the large panel of clinical and behavioural ASD features. We estimated that 107 children with data for at least three timepoints of the follow-up will be necessary to successfully conduct these analyses.

Inclusion of 1000 children at baseline, even considering an attrition rate of $10 \%$ commonly observed in ASD cohorts, ${ }^{20}$ exceeds the minimal subject number required to achieve the first objective and will provide sufficient power to investigate the heterogeneity of factors (sociodemographic, family, comorbidities and diagnostic factors) associated with the preidentified trajectories within the entire population and in each childhood age group, for each gender and according to each ASD primary diagnosis, by multivariate analysis. Indeed, we estimated a minimal sample size of 250 participants to perform relevant multivariate analyses according to (1) the number of available covariates (approximately 25) and (2) the recommendation of at least 10 participants for one single predictor. ${ }^{64}$ Finally, our objective is to obtain the largest sample possible to allow us to study the outcome according to criteria such as age and clinical subgroup. For a statistical power of $80 \%$ with an $\alpha$ at 0.05 , a sample size of 1000 children is targeted to allow assessment of the determinants of the natural history of ASD and its clinical heterogeneity and the examination of the prognostic factors.

The report of this study will be prepared in accordance with the guidelines set by the Strengthening the Reporting of Observational Studies in Epidemiology statement for observational studies. ${ }^{65}$ Data will be collected and analysed by confirmed statisticians of the ELENA study. $\mathrm{X}^{2}$ tests (for categorical variables), Student's t-tests and Fisher's tests (for normally distributed continuous variables) and Mann-Whitney U/Kruskal-Wallis tests (for non-normally distributed continuous variables) will be used for the description of characteristics and their comparison based on the primary ASD diagnosis. Missing data will be analysed and multiple imputation performed if required. Longitudinal relationships between risk factors and identified trajectories will be studied using multivariable linear and logistic regression models to assess factors associated with severity and specific long-term adaptive trajectories of ASD in childhood, based on the primary ASD diagnosis/ phenotype.

\section{Methodological considerations}

The primary limitation of this study is loss to follow-up and missing data points that would challenge the internal validity of the reported results of ELENA. However, our research team has extensive experience in achieving high follow-up rates in similar studies of long-term follow-up of patients. ${ }^{20}$ Efforts to minimise loss to follow-up will include respecting the time commitment of families and the use of formal tracking procedures of enrolled families, including obtaining multiple contacts for arranging follow-up and the strong interpersonal skills of the study staff. 


\section{Ethics and dissemination}

The study poses no risk to participants and their families. Signed informed consent is obtained from all participating families. Participation in the study does not interfere with the typical care patients receive in the autism centre. Clinical follow-up is provided by clinicians in the centres and, if necessary, participants are referred for additional clinical management (eg, rehabilitation, clinical psychology). Results from this study will be disseminated at national and international conferences and in peer-reviewed journals.

\section{Significance and outlook}

The study will provide indepth insight into the heterogeneity of the clinical presentation of ASD and associated factors. The prospectively collected clinical data will allow us to describe the outcome trajectories of ELENA participants for the 6 years following their inclusion and their determinants. The study results will inform clinicians, patients and their families of the expected course of ASD severity during childhood based on the ASD characteristics obtained at the time of diagnosis. A detailed understanding of the importance of perinatal factors, socioenvironmental factors, comorbidities, early medical care and medical care of the mother during pregnancy will assist in targeting patients at high risk of unfavourable behaviours and clinical ASD trajectories, who are the most likely to benefit from specific interventions. This strategy is essential to guide optimal clinical care and follow-up of children with ASD.

In addition, we will investigate the prevalence of a broad autistic phenotype and psychiatric/neurodevelopmental disorders among siblings of ASD, as we are regularly collecting information about siblings in our database.

Prenatal and perinatal occupational and residential histories will be collected through a web-based questionnaire tailored from early life exposure assessment tool (ELEAT) (https://eleat.ucdavis.edu/). Then, personal exposure to particulate matter (PM10, PM2.5) will be achieved through aerosol optical depth daily measurements by satellite images (resolution $1 \mathrm{~km}$ ) combined with other geographical data and calibrated with air quality monitoring station measurements. Personal exposure to pesticides will be done using geographical information systems and land use data (or public data from agricultural statistics) to determine the proximity and the type of crops within a $1 \mathrm{~km}$ radius around each home's address. A pesticides matrix will convert the crops exposure levels into pesticide exposure levels for each family of pesticides. The challenge is to determine the role of pesticides and air pollution in the aetiology of ASD, in comparison with children without ASD from a large French birth cohort french longitudinal study of children (ELFE), as well as in the ASD severity and trajectories.

In addition, a biobank is intended, comprising blood samples from the antecubital vein and droplets from fingertips on dried blood spot (DBS) collected by experienced nurses during a centre visit. Genetic and epigenetic studies are scheduled across three tasks: (1) toidentify simple genetic abnormalities (such as monogenic Mendelian or simple digenic forms), (2) to understand complex genetic forms architecture, including digenic, oligogenic or polygenic multifactorial models, and (3) to highlight epigenetic profiles on patient blood DNA. Patient exome sequencing and methylation profile on Illumina's EPIC chip will be analysed by the National Center for Research in Human Genomics. Bioinformatics analysis will be performed in collaboration with a molecular genetics laboratory, first on a $400 \mathrm{ASD}$ genes panel, and if no mutation would be found on the whole online mendelian inheritance in man (OMIM) genes then complex genetic form architecture will be studied in collaboration with a bioinformatics company.

\section{Author affiliations}

${ }^{1}$ Department of Psychiatry and Autism Resources Center, Montpellier University and University Hospital (CHU) of Montpellier, Montpellier, France

${ }^{2}$ U1178, INSERM, Centre de recherche en Epidemiologie et Sante des Populations, Villejuif, France

${ }^{3}$ U1198, MMDN, University of Montpellier, EPHE, INSERM, Montpellier, France

${ }^{4}$ Department of Child and Adolescent Psychiatry, Centre Hospitalier le Vinatier, Bron, France

${ }^{5}$ Centre de Ressources autisme Rhône-Alpes, CH Le Vinatier, Bron, France ${ }^{6}$ UMR 5292, Centre de Recherche en Neurosciences de Lyon (CNRS), Lyon, France ${ }^{7}$ Service de Psychiatrie de L'Enfant et de l'Adolescent, Pole Psychiatrie, Santé Mentale et Addictologie, Centre Ressources Autisme, Hopitaux Universitaires de Strasbourg, Strasbourg, France

${ }^{8}$ Service de Pédopsychiatrie, Hôpital Necker Enfants Malades, Assistance Publique Hopitaux de Paris, Paris, France

${ }^{9}$ Service Universitaire de Psychiatrie de l'Enfant et de l'Adolescent, Centre Hospitalier de Versailles, Le Chesnay, France

${ }^{10}$ EA 4047 HANDIReSP, Université de Versailles Saint-Quentin-en-Yvelines, Versailles, France

${ }^{11}$ Child and Adolescent Psychiatry Department, University Hospital CHU-Lenval, Nice, France

${ }^{12}$ Service Universitaire de Psychiatrie de l'Enfant et de l'Adolescent, Centre de Ressources Autisme Midi-Pyrénées, CHU de Toulouse, Toulouse, France

${ }^{13}$ Department of Medical Information, University Research and Hospital Center (CHU) of Montpellier, Montpellier, France

Acknowledgements We would like to thank all the participants of the study and their families for their long-lasting interest and the time they have spent and the efforts they have made for the study. We are thankful to the many staff members and clinicians who have contributed to recording the data, especially Florine Dellapiazza, Flore Couty, Myriam Soussana, Christelle Vernhet and Ela Miniarikova.

Collaborators ELENA Study Group: Professor Amaria Baghdadli, Dr Catherine Chabaux, Dr Clarisse Chatel, Professor David Cohen, Dr Emmanuel Damville, Dr Marie-Maude Geoffray, Professor Ludovic Gicquel, Professor Renaud Jardri, Dr Thierry Maffre, Dr Alexandre Novo, Dr Roxane Odoyer, Dr Marie-Joëlle Oreve, Dr Didier Périsse, Professor François Poinso, Dr Julien Pottelette, Dr Laurence Robel, Professor Catherine Rolland, Dr Marie Schoenberger, Dr Sylvie Serret, Dr Sandrine Sonié, Professor Mario Speranza and Dr Stéphanie Vespérini.

Contributors $A B$ conceived the study and drafted the manuscript. CR, CM, M-CP, $\mathrm{JL}$ and TA contributed to the conception of the research questions and protocol, critically reviewed and provided comments on the manuscript drafts, and agreed on the final submitted version. MS, M-MG, SS, LR, JP, TM, SV, BF, MM, ST and SM provided comments on the manuscript draft and agreed on the final submitted version.

Funding This research received support from the French Health Ministry (DGOS) and CNSA. Additional support was provided by the CHU of Montpellier (AOI).

Competing interests None declared.

Patient consent for publication Not required. 
Ethics approval The study and informed consent procedure have been approved by the Ethics Committee on the Research of Human Subjects at Marseille Mediterranean, France.

Provenance and peer review Not commissioned; externally peer reviewed.

Open access This is an open access article distributed in accordance with the Creative Commons Attribution Non Commercial (CC BY-NC 4.0) license, which permits others to distribute, remix, adapt, build upon this work non-commercially, and license their derivative works on different terms, provided the original work is properly cited, appropriate credit is given, any changes made indicated, and the use is non-commercial. See: http://creativecommons.org/licenses/by-nc/4.0/.

\section{REFERENCES}

1. Christensen DL, Bilder DA, Zahorodny W, et al. Prevalence and Characteristics of Autism Spectrum Disorder Among 4-Year-Old Children in the Autism and Developmental Disabilities Monitoring Network. J Dev Behav Pediatr 2016;37:1-8.

2. American Psychiatric Association. Diagnostic and statistical manual of mental disorders (DSM-5®). Washington D.C: American Psychiatric Publishing, 2013.

3. World Health Organization. The $I C D-10$ classification of mental and behavioural disorders: diagnostic criteria for research. Geneva: World Health Organization, 1993.

4. Chaste P, Leboyer M. Autism risk factors: genes, environment, and gene-environment interactions. Dialogues Clin Neurosci 2012;14:281-92.

5. Modabbernia A, Velthorst E, Reichenberg A. Environmental risk factors for autism: an evidence-based review of systematic reviews and meta-analyses. Mol Autism 2017;8:13.

6. Fein D, Barton M, Eigsti IM, et al. Optimal outcome in individuals with a history of autism. J Child Psychol Psychiatry 2013;54:195-205.

7. Howlin P, Goode S, Hutton J, et al. Adult outcome for children with autism. J Child Psychol Psychiatry 2004;45:212-29.

8. Gillberg C, Billstedt E. Autism and Asperger syndrome: coexistence with other clinical disorders. Acta Psychiatr Scand 2000;102:321-30.

9. Billstedt E, Gillberg IC, Gillberg C. Autism in adults: symptom patterns and early childhood predictors. Use of the DISCO in a community sample followed from childhood. J Child Psychol Psychiatry 2007;48:1102-10.

10. Vivanti G, Kasari C, Green J, et al. Implementing and evaluating early intervention for children with autism: Where are the gaps and what should we do? Autism Res 2018;11.

11. Baghdadli A, Pry R, Michelon C, et al. Impact of autism in adolescents on parental quality of life. Qual Life Res 2014;23:1859-68.

12. Ingersoll B, Hambrick DZ. The relationship between the broader autism phenotype, child severity, and stress and depression in parents of children with autism spectrum disorders. Res Autism Spectr Disord 2011;5:337-44.

13. Mugno D, Ruta L, D'Arrigo VG, et al. Impairment of quality of life in parents of children and adolescents with pervasive developmental disorder. Health Qual Life Outcomes 2007;5:22.

14. Rattaz C, Michelon C, Roeyers H, et al. Quality of Life in Parents of Young Adults with ASD: EpiTED Cohort. J Autism Dev Disord 2017;47:2826-37.

15. Horlin C, Falkmer M, Parsons R, et al. The cost of autism spectrum disorders. PLoS One 2014;9:e106552.

16. Masi A, DeMayo MM, Glozier N, et al. An Overview of Autism Spectrum Disorder, Heterogeneity and Treatment Options. Neurosci Bull 2017;33:183-93.

17. Constantino JN. Deconstructing autism: from unitary syndrome to contributory developmental endophenotypes. Int Rev Psychiatry 2018;30:18-24.

18. Geschwind DH, Levitt P. Autism spectrum disorders: developmental disconnection syndromes. Curr Opin Neurobiol 2007;17:103-11.

19. Fountain C, Winter AS, Bearman PS. Six developmental trajectories characterize children with autism. Pediatrics 2012;129:e1112-e1120.

20. Baghdadli A, Assouline B, Sonié S, et al. Developmental trajectories of adaptive behaviors from early childhood to adolescence in a cohort of 152 children with autism spectrum disorders. J Autism Dev Disord 2012;42:1314-25.

21. Baghdadli A, Picot MC, Michelon C, et al. What happens to children with PDD when they grow up? Prospective follow-up of 219 children from preschool age to mid-childhood. Acta Psychiatr Scand 2007;115:403-12.

22. Varcin KJ, Nelson CA. A developmental neuroscience approach to the search for biomarkers in autism spectrum disorder. Curr Opin Neurol 2016;29:123-9.
23. Øien RA, Schjølberg S, Volkmar FR, et al. Clinical Features of Children With Autism Who Passed 18-Month Screening. Pediatrics 2018;141:e20173596.

24. Stoltenberg C, Schjølberg S, Bresnahan M, et al. The Autism Birth Cohort: a paradigm for gene-environment-timing research. $\mathrm{Mol}$ Psychiatry 2010;15:676-80.

25. Charman T, Loth E, Tillmann J, et al. The EU-AIMS Longitudinal European Autism Project (LEAP): clinical characterisation. Mol Autism 2017:8:27.

26. Flanagan HE, Smith IM, Vaillancourt T, et al. Stability and Change in the Cognitive and Adaptive Behaviour Scores of Preschoolers with Autism Spectrum Disorder. J Autism Dev Disord 2015;45:2691-703.

27. Zaidman-Zait A, Mirenda P, Duku E, et al. Examination of bidirectional relationships between parent stress and two types of problem behavior in children with autism spectrum disorder. J Autism Dev Disord 2014;44:1908-17.

28. Georgiades S, Bishop SL, Frazier T. Editorial Perspective: Longitudinal research in autism - introducing the concept of 'chronogeneity'. J Child Psychol Psychiatry 2017;58:634-6.

29. HAS (Haute Autorité de Santé). Autisme et autres troubles envahissants du développement: interventions éducatives et thérapeutiques coordonnées chez l'enfant et l'adolescent. 2012 https://www.has-sante.fr/portail/jcms/c_953959/fr/autisme-etautres-troubles-envahissants-du-developpement-interventionseducatives-et-therapeutiques-coordonnees-chez-I-enfant-et-Iadolescent.

30. Baghdadli A, Aussilloux C, psychiatrie Fédération française de (FFP). Haute Autorité de Santé. Recommandations pour la pratique professionnelle du diagnostic de l'autisme. $2005 \mathrm{http}: / / \mathrm{www}$. hassante. fr/portail/jcms/c_468812/fr/recommandations-pour-lapratique-professionnelledu-diagnostic-de-l-autisme.

31. Bölte S, Poustka F. The relation between general cognitive level and adaptive behavior domains in individuals with autism with and without co-morbid mental retardation. Child Psychiatry Hum Dev 2002;33:165-72.

32. Perry A, Flanagan HE, Dunn Geier J, et al. Brief report: the Vineland Adaptive Behavior Scales in young children with autism spectrum disorders at different cognitive levels. J Autism Dev Disord 2009;39:1066-78.

33. Scattone D, Raggio DJ, May W. Comparison of the Vineland Adaptive Behavior Scales, Second Edition, and the Bayley Scales of Infant and Toddler Development, Third Edition. Psychol Rep 2011;109:626-34.

34. Bal VH, Kim SH, Cheong D, et al. Daily living skills in individuals with autism spectrum disorder from 2 to 21 years of age. Autism 2015;19:774-84.

35. Lord C, Rutter M, DiLavore P, et al. Autism diagnostic observation schedule-Second edition (ADOS-2). Los Angeles, CA: Western Psychological Services, 2012.

36. Rutter M, Lord C, Le Couteur A. Autism Diagnostic Interview-Revised (ADI-R). Los Angeles, CA: Western Psychological Services, 2003.

37. Sparrow SS, Cicchetti DV, Balla DA, et al. Vineland adaptive behavior scales: Survey forms manual. Circle Pines, MN: American Guidance Service, 2005.

38. HAS (Haute Autorité de Santé). Trouble du spectre de l'autisme: Signes d'alerte, repérage, diagnostic et évaluation chez l'enfant et adolescent [en ligne]. 2018 https://www.has-sante.fr/portail/upload/ docs/application/pdf/2018-02/trouble_du_spectre_de_lautisme_de_ lenfant_et_ladolescent_recommandations.pdf.

39. Lord C, Rutter M, DiLavore P, et al. Autism diagnostic observation schedule: ADOS: Manual. Los Angeles, CAT: Western Psychological Services, 2002.

40. Howlin P, Savage S, Moss P, et al. Cognitive and language skills in adults with autism: a 40-year follow-up. J Child Psychol Psychiatry 2014;55:49-58.

41. Wechsler D. Wechsler intelligence scale for children. New York: Psychological Corporation, 1949.

42. Wechsler D. Manual for the Wechsler preschool and primary scale of intelligence. New York: Psychological Corporation, 1967.

43. Wechsler D. Manual for the Wechsler intelligence scale for children, revised. New York, NY: The Psychological Corporation, 1974.

44. Wechsler D. WISC-IV: Administration and Scoring Manual. San Antonio, TX: Psychological Corporation, 2003.

45. Wechsler D. WPPSI-IV - Echelle d'intelligence de Wechsler pour enfants. Montreuil: ECPA-Pearson, 2014.

46. Wechsler D. WISC-V: Administration and Scoring Manual. San Antonio, Tex: NCS Pearson, Incorporated, 2014.

47. Kaufman AS, Kaufman NL. K-ABC: Kaufman assessment battery for children: Interpretive manual. Circle Pines, Minn: American Guidance Service, 1983. 
48. Schopler E. Individualized assessment and treatment for autistic and developmentally disabled children: Psychoeducational Profile Revised (PEP-R). 1. Austin, TX: PRO-ED, 1990.

49. Brunet O, Lézine I. Echelle de développement psychomoteur de la première enfance. Paris: EAP, 1981.

50. Josse D. Brunet-Lézine Révisé: Echelle de développement psychomoteur de la première enfance. Issy-les-Moulineaux: Éd. et applications psychologiques, 1997.

51. Dunn LM, Thériault-Whalen CM, Dunn LMÉchelle de vocabulaire en images Peabody: EVIP Psycan Toronto 1993.

52. Dunn LM, Dunn LM. PPVT: Peabody picture vocabulary test-revised : manual for forms $L$ and $M$. Circle Pines (Minnesota) : American guidance Service, 1981.

53. Ravens-Sieberer U, Auquier P, Erhart M, et al. The KIDSCREEN-27 quality of life measure for children and adolescents: psychometric results from a cross-cultural survey in 13 European countries. Qual Life Res 2007;16:1347-56.

54. Cousson F, Bruchon-Schweitzer M, Quintard B, et al. Analyse multidimensionnelle d'une échelle de coping: validation française de la WCC (ways of coping checklist). Psychologie française 1996;41:155-164.

55. Abidin R. Parenting Stress Index: Professional manual. Odessa, FL: Psychological Assessment Resources, 1995.

56. Zigmond AS, Snaith RP. The hospital anxiety and depression scale. Acta Psychiatr Scand 1983;67:361-70.
57. Achenbach TM, Edelbrock CS. Manual for the child behavior checklist and revised child behavior profile. Burlington,VT: University of Vermont, Department of Psychiatry, 1983.

58. Achenbach TM, Rescorla LA. ASEBA school-age forms \& profiles: An integrated system of multi-informant assessment. Aseba Burlington, VT 2001.

59. Achenbach TM, Rescorla LA. ASEBA preschool forms \& profiles: An integrated system of multi-informant assessment. Burlington, VT: University of Vermont, Research center for children, youth, \& families, 2000.

60. Aman MG, Singh NN, Stewart AW, et al. Psychometric characteristics of the aberrant behavior checklist. Am J Ment Defic 1985;89:492 502.

61. Dunn W. Sensory profile: User's manual. San Antonio, Tex: Psychological Corporation, 1999.

62. Andruff H, Carraro N, Thompson A, et al. Latent Class Growth Modelling: A Tutorial. Tutor Quant Methods Psychol 2009;5:11-24.

63. Nagin D. Group-based modeling of development. Cambridge, MA and London, England: Harvard University Press, 2005.

64. Gillaizeau F, Grabar S. Modèles de régression multiple. Sang Thrombose Vaisseaux 2011;23(7):360-70.

65. von Elm E, Altman DG, Egger M, et al. The Strengthening the Reporting of Observational Studies in Epidemiology (STROBE) statement: guidelines for reporting observational studies. PLoS Med 2007;4:e296. 